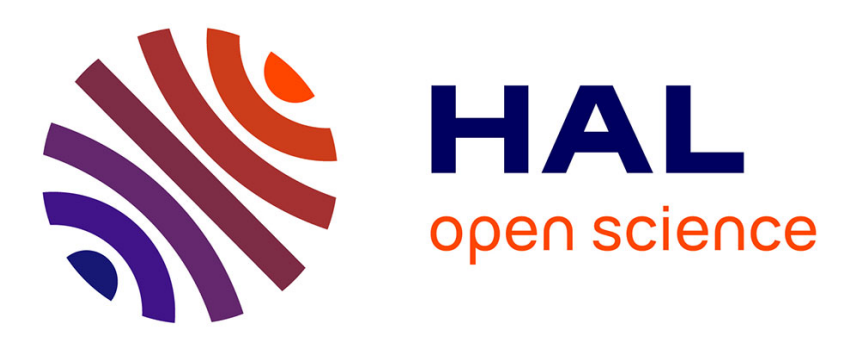

\title{
The cyclic ground state structure of the HF trimer revealed by far infrared jet-cooled Fourier transform spectroscopy.
}

Pierre Asselin, P. Soulard, B. Madebène, M. Goubet, T. R. Huet, Robert Georges, O. Pirali, Pascal Roy

\section{To cite this version:}

Pierre Asselin, P. Soulard, B. Madebène, M. Goubet, T. R. Huet, et al.. The cyclic ground state structure of the HF trimer revealed by far infrared jet-cooled Fourier transform spectroscopy.. Physical Chemistry Chemical Physics, 2014, 16 (10), pp.4797-4806. 10.1039/c3cp55047h . hal-00944403

\section{HAL Id: hal-00944403 https://hal.science/hal-00944403}

Submitted on 16 Sep 2014

HAL is a multi-disciplinary open access archive for the deposit and dissemination of scientific research documents, whether they are published or not. The documents may come from teaching and research institutions in France or abroad, or from public or private research centers.
L'archive ouverte pluridisciplinaire HAL, est destinée au dépôt et à la diffusion de documents scientifiques de niveau recherche, publiés ou non, émanant des établissements d'enseignement et de recherche français ou étrangers, des laboratoires publics ou privés. 


\title{
The cyclic ground state structure of HF trimer revealed by far infrared jet-cooled Fourier transform spectroscopy
}

\author{
P. Asselin, ${ }^{\mathrm{a}, \mathrm{b}^{*}}$ P. Soulard, ${ }^{\mathrm{a}, \mathrm{b}}$ B. Madebène $e^{\mathrm{a}, \mathrm{b}}$ \\ ${ }^{a}$ Sorbonne Universités, UPMC UnivParis 06, UMR 8233, MONARIS, F-75005, Paris, France \\ ${ }^{\mathrm{b}}$ CNRS, UMR 8233, MONARIS, F-75005, Paris, France \\ M. Goubet, ${ }^{\mathrm{c}}$ T.R. Huet ${ }^{\mathrm{c}}$ \\ ${ }^{\mathrm{c}}$ Laboratoire de Physique des Lasers, Atomes et Molécules, Bâtiment P5, UMR8523 \\ Université Lille 1-CNRS, F-59655 Villeneuve d'Ascq Cedex, France \\ R. Georges ${ }^{\mathrm{d}}$ \\ ${ }^{\mathrm{d}}$ Institut de Physique de Rennes, Campus de Beaulieu, Bat 11C, UMR 6251, Université de \\ Rennes 1-CNRS, 35042 Rennes Cedex, France \\ O. Pirali, ${ }^{\mathrm{e} f}$ P. Roy ${ }^{\mathrm{e}}$ \\ ${ }^{\mathrm{e}}$ Ligne AILES- Synchrotron SOLEIL, L'Orme des Merisiers, F-91192 Gif-sur-Yvette cedex, \\ France. \\ ${ }^{\mathrm{f}}$ Institut des Sciences Moléculaires d’Orsay, CNRS, Bat. 210, Université Paris-Sud, 91405 \\ Orsay, France.
}

\begin{abstract}
The rovibrationally resolved Fourier transform (FT) far infrared (FIR) spectra of two intermolecular librations of $(\mathrm{HF})_{3}$, namely the in-plane $v_{6}$ and out-of-plane $v_{4}$ bending fundamentals centered respectively at about $494 \mathrm{~cm}^{-1}$ and $602 \mathrm{~cm}^{-1}$, have been measured for the first time underjet-cooled conditions using the supersonic jet of the Jet-AILES apparatus. The simultaneous rotational analysis of 245 infrared transitions belonging to both bands enabled to determine the ground state (GS), $v_{6}$ and $v_{4}$ rotational and centrifugal distortion constants. These results brought definite experimental answers to the structure of such a weakly bound trimer: firstly the vibrationally averaged planarity of cyclic $(\mathrm{HF})_{3}$, also supported by the very small value of the inertia defect obtained in the GS, secondly the slight weakening of the hydrogen bond in the intermolecular excited states evidenced from the center of mass separations of the HF constituents determined in the ground, $\mathrm{v}_{6}=1$ and $\mathrm{v}_{4}=1$ states of $(\mathrm{HF})_{3}$ as well as the decrease of the fitted rotational constants upon excitation. Finally, lower bounds of about 2 ns on $v_{6}$ and $v_{4}$ states lifetimes could be derived from the deconvolution of experimental linewidths. Such long lifetimes highlight the interest to probe low frequency intermolecular motions of molecular complexes to get rid of constraints related to the vibrational dynamics of coupled anharmonic vibrations at higher energy, resulting in loss of rotational information.
\end{abstract}

"Author to whom correspondance should be addressed. Electronic mail:pierre.asselin@upmc.fr. 


\section{1- Introduction}

Hydrogen bonded dimers and trimers represent the simplestprototype systems for the study of pairwise additivity aspects and non-pairwise interactions such as three-body intermolecular forces which play a crucial role in the condensed phases of matter. ${ }^{1-6}$ In this context, considerable research has been dedicated to $(\mathrm{HF})_{\mathrm{n}}$ oligomers in the vapor phase providing important progress in the field of ab initio methods to derive potential energy surfaces and vibrational dynamics properties of small hydrogen fluoride clusters ${ }^{7-26}$, as well as in the various spectroscopic techniques used to record spectraof clusters. ${ }^{27-48}$

Successive microwave $e^{28,29}$ and infrared ${ }^{30-33}$ (IR) studies have been reported about $(\mathrm{HF})_{2}$ and (DF) $)_{2}$ during the 80's and 90's. The first high resolution (HR) spectra of these hydrogen bonded complexes enabled access to the dimer structure, dissociation energy, predissociation lifetimes and tunneling dynamics, and ultimately permitted to build reliable intermolecular potential energy surfaces for such four-atom clusters.4,49,50

In contrast to the large number of hydrogen bonded dimer studies reported up to now, only a few homomolecular trimers such as $(\mathrm{HCCH})_{3},{ }^{51}(\mathrm{HCN})_{3},{ }^{52}(\mathrm{HF})_{3}, 9^{36}(\mathrm{HCl})_{3},{ }^{53}$ $\left(\mathrm{CO}_{2}\right)_{3},{ }^{54,55}(\mathrm{DF})_{3},{ }^{41}$ and $\left(\mathrm{H}_{2} \mathrm{O}\right)_{3}{ }^{56}$ have been studied in nearly isolated gas phase supersonic jet environments associated to near or far infrared (FIR) tunable laser spectroscopy. Apart from $(\mathrm{HF})_{3}$, molecular constants could be obtained from rotationally resolved vibrational spectra. In the case of $\left(\mathrm{CO}_{2}\right)_{3}$, the assignment of the spectrum was facilitated by considering nuclear spin statistics. The donor stretching modes recorded for $(\mathrm{HCl})_{3}$ and $(\mathrm{DF})_{3}$ have pointed out the problems related to the analysis of higher energy bands: depending on the high density of levels and the strength of the coupling with the bath of states via intramolecular vibrational redistribution (IVR) a loss of fully resolved structure due to significant lifetime homogeneous broadening is likely to be observed, which is indeed large for $(\mathrm{DF})_{3}(4 \mathrm{GHz})^{41}$ but much smaller for $(\mathrm{HCl})_{3}(\leq 90 \mathrm{MHz}){ }^{53}$ Nevertheless, spectral analysis from such experiments provided reliable information about the structure and dynamics of trimers.

This is not the case of the hydrogen fluoride trimer and larger clusters for which available experimental IR data beyond cryogenic matrix spectra are very scarce $9^{36,41,57}$. Among these, mass selected vibrational predissociation spectra about the HF stretching bands of $(\mathrm{HF})_{3}$ and its isotopomers $9^{36}$ validated a planar and cyclic equilibrium structure in the $\mathrm{C}_{3 \mathrm{~h}}$ point group. Nevertheless, the fact remains that a definite experiment for deriving the vibrationally averaged structure of $(\mathrm{HF})_{3}$ is still missing. Previous high resolution infrared studies failed to provide structural information. IR-IR double resonance experiments within the very narrow frequency range of the $\mathrm{CO}_{2}$ laser enabled to observe two rovibrational transitions assigned to an in-plane overtone and an out-of-plane combination of torsional modes of $(\mathrm{HF})_{3} .9 \mathrm{No}$ homogeneous broadening was detected for these transitions $(\approx 15 \mathrm{MHz})$ around $950 \mathrm{~cm}^{-1}$, in contrast with the huge linewidths $(\approx 8 \mathrm{GHz})$ evidenced for the $3712 \mathrm{~cm}^{-1}$ HF stretching mode even at low temperature in HR jet-cooled spectra, due to IVR broadening.

The absence of permanent dipole moment for $(\mathrm{HF})_{\mathrm{n}}$ clusters $^{27}$ eliminates the possibility of a high resolution study in themicrowave spectral region. Consequently, high resolution far IR spectroscopy remains the only alternative to obtain structural information in 
the ground state, ${ }^{58}$ by a direct probing of the intermolecular modes of the trimer since the vibrational density of states isnegligibleat such low energies. Theoretical data available on $(\mathrm{HF})_{3}$ were used to guide our experimental strategy and to target the most favorable intermolecular probes.

Several ab initio investigations have predicted a cyclic $C_{3 h}$ equilibrium structure for $(\mathrm{HF})_{3},{ }^{18,26}$ confirming the experimental conclusions of Lisy et al. ${ }^{36}$ From the structures of several $(\mathrm{HF})_{\mathrm{n}}$ oligomers optimized at the coupled cluster level, dissociation energies, harmonic vibrational frequencies and IR intensities could be determined up to $n=4$. The large values of the equilibrium $\left(\mathrm{D}_{\mathrm{e}}\right)$ and corrected harmonic zero-point vibrational energy $\left(\mathrm{D}_{0}\right)$ dissociation energies, respectively calculated at 16 and $10.7 \mathrm{kcal} / \mathrm{mol}$ for $(\mathrm{HF})_{3}$, clearly underline the presence of three intermolecular bonds to form the cyclic structure (rather than two for a chain conformation). ${ }^{36,41,53,56}$ The character of the normal modes of (HF) $)_{n}$ oligomers can be classified according to group theory and vibrational coordinate analysis upon a planar oblate symmetric top having a $\mathrm{C}_{\mathrm{nh}}$ structure: $\mathrm{H}-\mathrm{F}$ intramolecular stretches in the 3300-3700 $\mathrm{cm}^{-1}$ range, $\mathrm{H}-\mathrm{F}$ intermolecular librations in the 500-1100 $\mathrm{cm}^{-1}$ range, F-F intermolecular stretches below $350 \mathrm{~cm}^{-1}$ and ring deformations below $100 \mathrm{~cm}^{-1}$.

Concerning $(\mathrm{HF})_{3}$, a previous harmonic vibrational analysis evidenced strong IR intensities $\left(\mathrm{I}_{6} \approx 700 \mathrm{~km} / \mathrm{mol}, \mathrm{I}_{4} \approx 410 \mathrm{~km} / \mathrm{mol}\right)$ for the two librational modes, the in-plane $\left(v_{6}, E^{\prime}\right)$ and out-of-plane $\left(v_{4}, A^{\prime \prime}\right)$ bending fundamentals, ${ }^{18}$ which gives hope for their experimental observation. Large anharmonic effects in such a cluster make uncertain the reliability of the predicted harmonic frequencies for the $v_{6}$ and $v_{4}$ modes, respectively at 603 and $702 \mathrm{~cm}^{-1}$, much higher than the values obtained from a $6 \mathrm{D}$ variational calculation $(540$ and $\left.637 \mathrm{~cm}^{-1}\right),{ }^{26}$ themselves higher than the only available experimental data about $v_{6}$ and $v_{4}$ modes in neon (argon) matrices, respectively at 477 and $590 \mathrm{~cm}^{-1}\left(446\right.$ and $\left.560 \mathrm{~cm}^{-1}\right){ }^{47,48}$ In summary, the presence of two intense bending modes separated by about $100 \mathrm{~cm}^{-1}$, in a spectral range where the vibrational density of states is low enough to preclude IVR broadening, is definitely favorable to record a rotationally resolved spectrum in jet-cooled conditions. We have undertaken a simultaneous study of the $v_{6}$ and $v_{4}$ modes using our JetAILES apparatus consisting in a continuous supersonic jet coupled to a high resolution Fourier Transform IR spectrometer (FTS). The multiplex advantage of FT spectroscopy could be fully exploited here to derive simultaneously ground state, $v_{6}$ and $v_{4}$ excited state parameters from a global least-squares fit and to bring new insights about the HF trimer in its vibrational ground state.

Section 2 gives a short description of the Jet-AILES apparatus implemented at SOLEIL and describes the spectra recorded in the mid infrared (MIR) and FIR regions relative to $\mathrm{HF}$ stretches and intermolecular librations, respectively. In Section 3 high level vibrational calculations relative to cyclic $(\mathrm{HF})_{3}$ are presented in support of the experimental data. Section 4 reports on the rotational analysis, global fit and predicted spectra of $v_{6}$ and $v_{4}$ bending modes. Structural data derived from the rovibrational analysis of the high resolution FIR Jet-AILES spectrum of $(\mathrm{HF})_{3}$ are finally discussed with the support of theoretical calculations. 


\section{2-Experimental and theoretical details}

\subsection{The Jet-AILES apparatus}

The Jet-AILES apparatusis a continuous supersonic high pressure jet coupled to the high resolution Fourier transform spectrometer (IFS $125 \mathrm{HR}$ Bruker, $0.001 \mathrm{~cm}^{-1}$ maximal resolution) associated to the AILES beamline of the synchrotron SOLEIL. It was recently described in details and only the main characteristics will be presented hereafter (Figure 1). ${ }^{59,60,61}$

The modulated IR parallel beam of the FTS is sent into an opticalcompartment connected to the back parallel exit port. A first setof two gold coated planar (M1) and toroidal (M2) mirrorsisfocusing the IR beam into the expansion chamber orthogonally to theslit-jet expansion with a magnification factor of 1.14 . The IR beam is thendirectly sent into the detector compartment in which a second set oftoroidal (M3) and planar (M4) mirrors is used to focus the IRbeam either onto an InSb detector for the HF stretches (MIR) or a bolometer for the intermolecular librations (FIR).

The expansion chamber is connected to an ensemble of Roots blowers backed by a dry primary pump delivering an effective pumping capacity of about $1800 \mathrm{~m}^{3} / \mathrm{h}$.The mechanical vibrations are efficiently damped on one hand by decoupling the pumping unit from the vacuum chamber with two dedicated bellows mounted on the pumping line, on the other handbymounting independently the three vacuum compartments on a heavy framework tightly fixed to the ground. The expansion chamber is isolated from the two other compartmentsby two optical windows (wedged $\mathrm{KBr}$ in the MIR or $60 \mu \mathrm{m}$ thick polypropylene films in the FIR). They are set asclose as possible to the slit nozzle in order to limit the IR absorption by the residual warm gas filling the experimental chamber after expansion. The optical and detector compartments are evacuatedtogether with the FTS in the $10^{-4} \mathrm{hPa}$ range in order to avoidatmospheric absorption.A series of slit nozzles of various fixed lengths (30, 60 and $90 \mathrm{~mm}$ ) andwidths (10 up to $300 \mu \mathrm{m})$ is available to investigate weakly bonded complexesand clustersin the gas phase.

The flows of $\mathrm{HF}$ and carrier gas are independentlyregulated by massflow controllers (Bronkhorst 0.02-1 standard liter/minutes (slm) model F-101D-1K0-RAD-33-Kand 2-100 slm model F-202AV, respectively). Paired liquid nitrogen $\left(\mathrm{LN}_{2}\right)$ traps connected in serieswere mounted between the Roots and the primary pumps to condensate corrosive acid vapoursand neutralized afterwards in a soda/water mixture.

Thestagnation pressure and the residual pressure were measuredusing absolute capacitance manometers (MKS bakeable Baratron5000 torr model 615A and MKS Baratron 100 torr model 626A,respectively).

\subsection{The Jet-AILES MIR spectra}

Hydrogen fluoride (Messer, electronic quality) was used without any further purification. Argon was preferred to helium as carrier gas to enhance the formation of small $(\mathrm{HF})_{\mathrm{n}}$ homomolecular clusters. 
As a first step, the absorption signal of the HF trimer was optimized in the HF stretching region with respect to those of the dimer and larger clusters. A series of medium resolution jet-cooled FT spectra $\left(0.1 \mathrm{~cm}^{-1}, 100\right.$ co-added scans) have been recorded to test different HF:Ar dilutions, slit geometries and stagnation pressures. For these experiments, we used a tungsten lamp, a $\mathrm{KBr}$ beamsplitter and a InSb detector equipped with a band pass filter centered at $3650 \mathrm{~cm}^{-1}$, with a $400 \mathrm{~cm}^{-1}$ full width at half maximum (FWHM) and positioned in the detector compartment. The strongest absorptions have been obtained with a $90 \mathrm{~mm} \times$ $100 \mu \mathrm{m}$ slit nozzle supplied with $5 \%$ HF diluted in a 20 slm mass flow rate of Ar. The corresponding stagnation and chamber pressures are $240 \mathrm{hPa}$ and $0.68 \mathrm{hPa}$, respectively.

A strong enhancement of the number of $(\mathrm{HF})_{3}$ and $(\mathrm{HF})_{4}$ oligomers has been observed when adding $\mathrm{CH}_{3}$ Clto the HF:Ar mixture. To date we have no firm explanation about the role of $\mathrm{CH}_{3} \mathrm{Cl}$ as third collision partner in the nucleation process, but as a matter of fact its presence clearly contributes to boost the formation of larger $(\mathrm{HF})_{\mathrm{n}}$ oligomers together with the $\mathrm{CH}_{3} \mathrm{Cl}$-HF dimers observed at $3777 \mathrm{~cm}^{-1}$ (Figure 2b,c). A similar effect has already been observed in $\mathrm{N}_{2} \mathrm{O}$ :HF supersonic expansions seeded with $\operatorname{argon}^{62}$. A small amount of $\mathrm{N}_{2} \mathrm{O}$ greatly enhanced the $(\mathrm{HF})_{2}$ dimer production with respect to that of $\mathrm{N}_{2} \mathrm{O}-\mathrm{HF}$. Cryogenic matrix experiments are planned to evidence the clusters formation during the codeposition of a $\mathrm{CH}_{3} \mathrm{Cl}: \mathrm{HF}$ :Ar mixture.

Figure 2 displays a series of Jet-AILES spectra recorded for different conditions of dilution of the $\mathrm{CH}_{3} \mathrm{Cl}$ :HF:Ar mixture. Without $\mathrm{CH}_{3} \mathrm{Cl}$ (Fig. 2a), (HF) $)_{2}$ and $(\mathrm{HF})_{3}$ bonded stretch bands located respectively at 3868 and $3712 \mathrm{~cm}^{-1}$, are the only detectable absorptions with a trimer signal rather weak. The addition of $\mathrm{CH}_{3} \mathrm{Cl}$ shifts the size distribution of $(\mathrm{HF})_{\mathrm{n}}$ clusters to larger $\mathrm{n}$ : the intensity of the 3712 and $3447 \mathrm{~cm}^{-1}$ bands of $(\mathrm{HF})_{3}$ and $(\mathrm{HF})_{4}$ clusters increases substantially and then reaches a maximum for $\mathrm{CH}_{3} \mathrm{Cl}: \mathrm{HF}$ ratios respectively equal to one (Fig. 2b) and two (Fig. 2c). Broad absorptions are also observed at 3575, 3587, 3638 and $3747 \mathrm{~cm}^{-1}$ for the highest $\mathrm{CH}_{3} \mathrm{Cl}$ concentration (Fig. 2c).

\subsection{The Jet-AILES FIR spectra}

From the preliminary MIR study, the expansion conditions maximizing the absorption of the intermediate $(\mathrm{HF})_{3}$ cluster could be optimized with respect to $(\mathrm{HF})_{2}$ and $(\mathrm{HF})_{4}$ and consequently the 1:1:20 mixture was used (Fig. 2b).

For the FIR experiments, we used a $6 \mu \mathrm{m}$ thick Mylar beamsplitter and a liquid He cooled Si-bolometer detector equipped with a polyethylene filter limiting the spectral range to 50-650 $\mathrm{cm}^{-1}$. Survey Jet-AILES FIR spectra have been firstly recorded at 0.05 and $0.01 \mathrm{~cm}^{-1}$ medium resolutions (Figures $3 \mathrm{a}$ and $3 \mathrm{~b}$ ) to localize the band centers of the bending fundamental bands of $(\mathrm{HF})_{3}$. Secondly, globar and synchrotron radiation (SR) sources have been tested at higher resolution $\left(0.005 \mathrm{~cm}^{-1}\right)$ to compare their corresponding signal-to-noise $(\mathrm{S} / \mathrm{N})$ ratios. In the investigated spectral range, it appears that the globar source still keeps a slight advantage at this resolution. The $\mathrm{S} / \mathrm{N}$ rather favors the synchrotron radiation for lower frequencies (below $300 \mathrm{~cm}^{-1}$ ) and/or higher resolutions up to $0.001 \mathrm{~cm}^{-1}$. Note finally that the choice of the globar source for these experiments owed to the fact that the SR radiation saturated the Si-bolometer for apertures larger than $1.3 \mathrm{~mm}$ even for the lowest available stage of gain preamplification. A reduction of the gain preamplification is planned in further experiments. 
The Jet-AILES FIR spectrum used for the rovibrational analysis of both bending intermolecular modes of cyclic $(\mathrm{HF})_{3}$ is displayed in Figure 3c. It represents the averaging of 700 scans recorded at $0.005 \mathrm{~cm}^{-1}$ resolution which required a maximal iris aperture of 3.15 $\mathrm{mm}$ corresponding to an IRspot diameter of about $3.6 \mathrm{~mm}$ focused at the center of the slit, 3 $\mathrm{mm}$ downstream from the exit plane of the nozzle.

Two fully resolved bands are observed at about 494 and $602 \mathrm{~cm}^{-1}$. On the basis of cryogenic matrix experiments ${ }^{47,48}$ and variational calculations, ${ }^{26}$ they are assigned to the inplane $\left(v_{6}, E^{\prime}\right)$ and out-of-plane $\left(v_{4}, A^{\prime \prime}\right)$ bending fundamental bands. Expectedly, calculated harmonic vibrational frequencies fall largely above gas phase values while the neon matrix observed ones are red shifted by about 2-4\% supporting futher the attribution.

The line position accuracy is a function of the $\mathrm{S} / \mathrm{N}$ ratio and was estimated to be 0.0005 $\mathrm{cm}^{-1}$ for the highest resolution spectrum.

\subsection{Computational details}

Second order Møller-Plesset calculations (MP2) were carried out using the Gaussian09 software package. ${ }^{63}$ Explicitly correlated coupled clusters (CCSD(T)-F12) calculations were carried out using the Molpro2010 package. $^{64}$ The frozen-core approximation was used throughout. Dunning and coworkers ${ }^{65,66}$ augmented correlation consistent basis set aug-cc-pVTZwas used (denoted hereafter AVTZ). All geometries optimizations were performed using "verytight" convergence criteria.

At the MP2 level, anharmonic correction on frequencies and geometrical parameters were calculated using thesecond-order vibrational perturbation theory (VPT2) as implemented in Gaussian09. ${ }^{67}$ Anharmonic correction have been extrapolated to the CCSD(T) level using eq (1) where $\omega$ and $v$ are the harmonic and anharmonic frequencies, respectively.

$$
v_{\mathrm{CCD}(\mathrm{T})}=\omega_{\mathrm{CCSD}(\mathrm{T})}-\left(\omega_{\mathrm{MP} 2}-v_{\mathrm{MP} 2}\right)
$$

\section{Theoretical results}

As prototype of hydrogen bond complexes, a lot of theoretical studies have been realized on small $(\mathrm{HF})_{\mathrm{n}}$ clusters and more particularly on $(\mathrm{HF})_{3}$, the simplest complex to analyze nonpairwise additivity. However most of those studies have neglected anharmonicity and only focused on equilibrium structure and harmonic frequencies (see ref [77] for a partial review of earlier work on harmonic frequencies). Xantheas ${ }^{68}$ computed VPT2 anharmonic frequencies for the trimer using MP2/aug-cc-pVDZ high order energy derivative, and compared its results with those of Chaban et $a l^{69}$ obtained using a correlation corrected vibrational self-consistent field approach on 2D grids potential energy surface (PES) computed at MP2/TZV level of theory. He concluded that both methods yield similar results, but the ab initio level used is too low to reach spectroscopic accuracy. Quack and Suhm built several semi-empirical PES for HF trimer based on the sum of manybody terms. ${ }^{76,77}$ Three body terms were determined from ab initio calculations only while two body terms were based on ab initio calculations but then empirically adjusted. Diffusion Quantum Monte-Carlo (DQMC) calculations have been performed by $\mathrm{Suhm}^{76}$ for a few modes of the trimer on the SNB+HF3BL PES. Wang and Carrington ${ }^{26}$ analyzed the same PES and the improved 
SO3+HF3BG PES using an elegant 6D variational approach which couples all the bending modes of the trimer. ${ }^{26}$ The results of both studies are reported in Table 1 . As they differ from our experimental results by several tens of $\mathrm{cm}^{-1}$, we decided to theoretically study $(\mathrm{HF})_{3}$ using an approach which showed its efficiency on the water trimer, ${ }^{70}$ by firstly computing the harmonic frequencies at a high level of theory and then reporting on those frequencies the anharmonic correction obtained from VPT2 at a lower level of theory.

We chose to use the CCSD(T)-F12 approach combined with the AVTZ basis set. As the convergence to complete basis set (CBS) limit is faster with the CCSD(T)-F12 approach than with $\operatorname{CCSD}(\mathrm{T})$, harmonic frequencies should be close to the $\operatorname{CCSD}(\mathrm{T}) / \operatorname{CBS}$ ones. The anharmonic corrections were computed at MP2/AVTZ level. As Wang and Carrington ${ }^{26}$ proved the absence of tunneling effect within the trimer, we can reasonably rely on the anharmonic correction obtained by the VPT2 approach. Both harmonic and anharmonic frequencies are reported in Table 1.

Due to the $\mathrm{C}_{3 \mathrm{~h}}$ symmetry of the complex (see Figure 4), only four vibrational modes are IR active: the asymmetric HF stretching $v_{5}$, the in-plane $v_{6}$ and out-of-plane $v_{4}$ bendings, and the asymmetric intermolecular stretching $v_{7}$. Our anharmonic frequencies are in excellent agreement with all observed gas phase frequencies of the trimer, which are reproduced with a maximum deviation of $2 \mathrm{~cm}^{-1}$. Because of its low intensity, the $v_{7}$ mode could not be observed in the gas phase. However our calculated frequency of $173 \mathrm{~cm}^{-1}$ also agrees well with the neon matrix value of $167 \mathrm{~cm}^{-1}$, taking into account that the neon matrix lowers $(\mathrm{HF})_{3}$ frequencies by a few wavenumbers with respect to the gas phase (Table 1).

We tried to understand whether the shift between Wang's frequencies ${ }^{26}$ and experiment were due to neglecting the coupling with stretching modes or came from an error on the PES used. The anharmonic frequencies $v_{6}$ and $v_{4}$ computed by Suhm (410(10) and $527(10) \mathrm{cm}^{-1}$ ) are far from the observed values, mainly due to errors on the two body terms of the SNB+HF3BL PES. However these DQMC calculations which take into account all the vibrational couplings can be used to estimate the error on the fundamentals computed by Wang in neglecting the coupling with the intra- and inter-molecular stretchings. $v_{6}$ and $v_{4}$ are both overestimated by $27(10) \mathrm{cm}^{-1}$ using the SNB+HF3BL PES. From our VPT2 calculation, the coupling between stretchings and $v_{6}$ or $v_{4}$ is respectively equal to -51 and $-46 \mathrm{~cm}^{-1}$. If we report those corrections on the frequencies of the SO3+HF3BG PES, we found 489 [513(10)] $\mathrm{cm}^{-1}$ for $v_{6}$ and 591 [610(10)] $\mathrm{cm}^{-1}$ for $v_{4}$, using respectively VPT2 [DQMC] corrections. The good agreement between corrected SO3+HF3BG PES and observed frequencies seems to confirm that the error made by the $6 \mathrm{D}$ variational approach mainly comes from neglecting the coupling with stretching modes, and not from the PES used.

FromIR-IR double resonance spectroscopy, Kolenbrander et al $^{9}$ observed two bands of the trimer at 940.5 and $954.4 \mathrm{~cm}^{-1}$, assigned respectively to the $2 v_{6}$ overtone and the $\left(v_{4}+v_{8}\right)$ combination, assuming in-plane and out-of-plane bending modes uncoupled. Wang et al. ${ }^{26}$ proposed that one of them could correspond to the $\left(v_{6}+v_{8}\right)$ combination computed at 956 and $983 \mathrm{~cm}^{-1}$. From our calculated frequencies, we list the combination bands $\left(v_{i}+v_{j}\right)$ and overtones $\left(2 v_{i}\right)$ which fall in the $900-1000 \mathrm{~cm}^{-1}$ range. Keeping only the IR active ones by symmetry $\left(E^{\prime}\right.$ and $\left.A^{\prime \prime}\right)$ only three transitions are remaining at $926\left(v_{2}+v_{7}\right), 948\left(2 v_{6}\right)$ and950 $\left(v_{4}+v_{8}\right) \mathrm{cm}^{-1}$. The $\left(v_{6}+v_{8}\right)$ combination is discarded because it falls below $900 \mathrm{~cm}^{-1}$. The 
$\left(v_{2}+v_{7}\right)$ combination band, never computed in earlier works has a frequency much lower than the observed frequencies and is discarded as well. As our calculations overestimate $v_{6}$ by $2 \mathrm{~cm}^{-1}$ and underestimate $v_{4}$ by $2 \mathrm{~cm}^{-1}$ with respect to observed frequencies, we can reasonably conclude that the corrected computed frequencies for $2 v_{6}$ and $\left(v_{4}+v_{8}\right)$ nicely match experimental ones observed by Kolenbrander. Moreover, an excellent agreement is obtained between computed and experimental anharmonic shifts, namely the $\left(2 v_{6}\right)-2\left(v_{6}\right)$ difference, respectively equal to -46 and $-49.0 \mathrm{~cm}^{-1}$.

On the grounds of the good results obtained in reporting anharmonic correction from VPT2 computed at MP2/AVTZ level on CCSD(T)-F12/AVTZ harmonic frequencies, the same approach was applied to the structural parameters. The CCSD(T)-F12/AVTZ equilibrium parameters are reported in Figure 4 and should be associated to the equilibrium rotational constants, i.e. $B_{e}=0.25422 \mathrm{~cm}^{-1}$ and $C_{e}=0.12711 \mathrm{~cm}^{-1}$. In Table 2 we reported $B$ and $C$ constants corrected from anharmonicity at $\operatorname{CCSD(T)-F12~level~using~the~anharmonic~}$ correction from VPT2 as $\mathrm{B}_{\text {anh }}^{\mathrm{CCSD}(\mathrm{T})}=\mathrm{B}_{\mathrm{e}}^{\mathrm{CCSD}(\mathrm{T})}-\left(\mathrm{B}_{\mathrm{e}}^{\mathrm{MP} 2}-\mathrm{B}_{\mathrm{anh}}^{\mathrm{MP} 2}\right)$ for the ground state, $v_{6}$ and $v_{4}$ states. The comparison with experimental rotational constants will be discussed in section 5 .

\section{Rovibrational analysis}

Experimental spectra of the cyclic hydrogen fluoride trimer were analyzed within the oblate symmetric rovibrator approximation including centrifugal distortion constants $D_{J}, D_{J K}$ and $D_{K}$. Ground state rotational energy levels are given by: ${ }^{71}$

$$
E(J, K)=B J(J+1)+(C-B) K^{2}-D_{J} J^{2}(J+1)^{2}-D_{J K} J(J+1) K^{2}-D_{K} K^{4}
$$

For the $v_{6}$ perpendicular band,rotational energy levels are calculated from the following expression which includes the first order rotation-vibration interaction: ${ }^{71}$

$$
\begin{gathered}
E\left(v_{6}, J, K\right)=v_{6}+B^{\prime} J(J+1)+\left(C^{\prime}-B^{\prime}\right) K^{2}-2 \zeta C^{\prime} K l-D_{J}^{\prime} J^{2}(J+1)^{2} \\
-D_{J K}^{\prime} J(J+1) K^{2}-D_{K}^{\prime} K^{4}
\end{gathered}
$$

where $\zeta$ is the first-order Coriolis constant for the degenerate vibration $v_{6}$. Transitions follow the selection rules $\Delta J=0, \pm 1 ; \Delta K= \pm 1 ; \Delta l= \pm 1$ and $\Delta|K-l|=0$. Rotational branches for the $v_{6}$ perpendicular band will be designated by ${ }^{p, r} P(J, K)^{p, r} Q(J, K)$ and ${ }^{p, r} R(J, K)$ where the superscripts $p, r$ correspond to $\Delta K=-1,+1$ and the capital letters $P, Q, R$ to $\Delta J=-1,0,1$,respectively.

For the $v_{4}$ parallel band, rotational energy levels are calculated from the following expression: ${ }^{71}$

$$
\begin{gathered}
E\left(v_{4}, J, K\right)=v_{4}+B^{\prime} J(J+1)+\left(C^{\prime}-B^{\prime}\right) K^{2}-D_{J}^{\prime} J^{2}(J+1)^{2} \\
-D_{J K}^{\prime} J(J+1) K^{2}-D_{K}^{\prime} K^{4}
\end{gathered}
$$


Transitions follow the selection rules $\Delta J=0, \pm 1 ; \Delta K=0$.Rotational branches for the $v_{4}$ parallel band will be designated by ${ }^{q} P(J, K),{ }^{q} Q(J, K)$ and ${ }^{q} R(J, K)$ where the superscript $q$ correspond to $\Delta K=0$. For both perpendicular and parallel bands, each branch is composed of a number of sub-bands with $J \geq K$. In what follows, the notation $\Delta X=\left(X^{\prime}-X\right) / X$ is used to designate the change in the rotational constant $X(=B, C)$ in an excited state.

The statistical weights for $(\mathrm{HF})_{3}$ depends on nuclear statistics for a complex which belongs to the $C_{3 h}$ symmetry group. The nuclear spins relevant to the $(\mathrm{HF})_{3}$ complex are $I_{H}=1 / 2$ and $I_{F}=1 / 2$. Statistical weights have been determined in the same manner as for $\left(\mathrm{C}_{2} \mathrm{H}_{2}\right)_{3}$ (which possesses two hydrogen atoms of spin $\left.1 / 2\right)^{72}$, one obtains for the GS state $g_{I}=48$ for $K=3 n$ and $g_{I}=40$ otherwise.

The SPFIT/SPCAT suite of programs, developed by Pickett, ${ }^{73}$ was used for the determination of the molecular spectroscopic parameters.

In contrast with previous infrared studies performed on cyclic trimers, the simultaneous detection of two fully resolved bands of vibration-rotation leads to a complete determination of the ground state constants $B, C, D_{J}, D_{J K}$ and $D_{K}$ as well as the excited state constants $B^{\prime}, C^{\prime}, D_{J}^{\prime}, D_{J K}^{\prime}$ and $D_{K}^{\prime}$. In the following section, the rotational analysis for each band are presented separately, together with the results derived from the global fit including the complete set of assigned lines belonging to both intermolecular bending modes.

\subsection{The jet-cooled in-plane bending $v_{6}$ band}

The rovibrational structure of the fundamental band $v_{6}$ of $(\mathrm{HF})_{3}$, which extends from 488 to $499 \mathrm{~cm}^{-1}$, is displayed in Figure 5a. A preliminary assignment was done by assuming a strictly planar ground state structure for the oblate top perpendicular band contour $(A=B=$ $2 C$ ). Starting from a prediction based on calculated ground state rotational and quartic centrifugal distortion rotational constants, the clusters of intense lines (see insert in Fig.3) spaced from 0.26 to $0.34 \mathrm{~cm}^{-1}$ on the low frequency side, and from 0.22 to $0.16 \mathrm{~cm}^{-1}$ on the high frequency side were assigned to ${ }^{p} P(J, K)$ and ${ }^{r} R(J, K)$ transitions with $J \geq K$, respectively. The frequency spacing increases on the ${ }^{p} P(J, K)$ side and decreases on the ${ }^{r} R(J, K)$ side which is explained by the differences between successive transitions of the type ${ }^{p} P(J+1, K+1)$ and ${ }^{p} P(J, K)$, or ${ }^{r} R(J, K)$ and ${ }^{r} R(J-1, K-1)$. These differences include the sum of a J-dependentterm almost equal to $2 B^{\prime}$ and K-dependent terms whose sign changes according to $\Delta K= \pm 1$.

The substructure within each cluster displays a characteristic pattern composed of typically four ${ }^{p} P(J, K)$ or ${ }^{r} R(J, K)$ lines equally spaced in frequency whatever the value of $J$. Such a pattern was assigned for a series of ${ }^{p} P(J-n, K-2 n)$ or ${ }^{r} R(J-n, K-2 n)$ lines with $n=0-3$.

Concerning the ${ }^{p, r} Q(J, K)$ lines, these transitions are expected to be significantly less intense than ${ }^{p} P(J, K)$ and ${ }^{r} R(J, K)$ ones. They are blended for large values of $J$ and $K$ but we could nevertheless identify some of them close to the band center for small values of $K .135$ lines have been finally assigned from which $62{ }^{p} P(J, K)$ lines, $62{ }^{r} R(J, K)$ lines, $4{ }^{p} Q(J, K)$ 
lines and $7{ }^{r} Q(J, K)$ lines. The highest rotational quantum number $J$ corresponds to 19 for ${ }^{p} P$ transitions and 17 for ${ }^{r} R$ transitions.

\subsection{The jet-cooled out-of-plane bending $v_{4}$ band}

The fundamental band $v_{4}$ of $(\mathrm{HF})_{3}$ is displayed in Figure 6a. Its rovibrational structure between 594 and $609 \mathrm{~cm}^{-1}$ shows the characteristic $P Q R$ structure of a parallel band with a $Q$ branch fully resolved and a resolved $K$ substructure for the ${ }^{q} P$ and ${ }^{q} R$ transitions. 110 lines have been assigned, namely $47{ }^{q} P(J, K)$ lines, $28{ }^{q} Q(J, K)$ lines and $35^{q} R(J, K)$ lines with highest values of $J$ equal to 14,21 and 12 for ${ }^{q} P^{q} Q$ and ${ }^{q} R$ transitions, respectively.

\subsection{Determination of molecular parameters}

Ground state and upper states parameters of $(\mathrm{HF})_{3}$ have been obtained by a global fitting procedure of the 245 IR transitions from both $v_{6}$ and $v_{4}$ bands. The simultaneous analysis of bending bands allows to reduce the correlations between several pairs of parameters, such as $B$ and $B^{\prime}, C$ and $C^{\prime}, C^{\prime}$ and $\zeta$, and so to determine them independently.

Regarding the global fit, all the rotational parameters were set free to test the robustness of our attributions. The ground state $D_{K}$ constant is the only one associated with a large standard deviation, likely due to correlation effects. In the following, this parameter is therefore fixed to its ab initio value. Finally, 16 rotational parameters ( 4 for the ground state and 6 for each upper state) were adjusted to reproduce the 245 values with a root mean square (RMS) value of $0.00131 \mathrm{~cm}^{-1}$. The Coriolis coupling constant deduced from the fit is undetermined and very small, which indicates a very little vibrational angular momentum in the excited $v_{6}$ band. The results of the least squares optimization are presented in Table II. All assigned lines, together with the observed minus calculated wavenumbers are provided as Electronic Supplementary Information in Table S1. Figures 5b and $6 \mathrm{~b}$ display a global view of simulated spectra of both bending in-plane $v_{6}$ and out-of-plane $v_{4}$ bands issued from our best fit.

All the simulated spectra were plotted using the PGOPHER program. ${ }^{74}$ A rotational temperature $\left(T_{\text {rot }}\right)$ of $30 \mathrm{~K}$ was found to provide the best agreement between the observed and calculated relative intensities of the rotational ${ }^{p, q, r} P, Q, R$ branches of both perpendicular and parallel bands. While the $25 \mathrm{~K}$ value of $T_{\text {rot }}$ obtained for the recent Jet-AILES spectra of naphthalene seeded in $\mathrm{He}$ is probably due to the $423 \mathrm{~K}$ nozzle temperature necessary to PAH vaporization $^{60}$, the jet-cooled conditions used here $\left(5 \% \mathrm{HF}\right.$ seeded in a $\mathrm{CH}_{3} \mathrm{Cl}$ :Ar mixture) represents our best compromise to maximize the production of small HF clusters using a heavy carrier gas instead $\mathrm{He}$ and to obtain a reasonably low value of $T_{\text {rot }}$.

The quality of the fit is illustrated by the comparison between observed and simulated spectra for a small portion of ${ }^{r} R(J, K)$ transitions of the $v_{6}$ band (Figure 7) and for the ${ }^{q} Q(J, K)$ transitions of the $v_{4}$ band (Figure 8). 


\section{Discussion}

Vibrationally averaged structural properties, which have been limited to a very few cyclic trimers so far, can be extracted from the results of the present rovibrational analysis of $(\mathrm{HF})_{3}$.

To check the consistency of our fit with respect to the state-of-the-art knowledge about the $(\mathrm{HF})_{3}$ structure, it is meaningful to verify the planarity of this trimer by exploiting indirect information obtainable from rotational parameters. Firstly, we used the relation between the distortion constants used by Han et al. ${ }^{53}$ to verify the planarity of $(\mathrm{HF})_{3}$. Indeed, an oblate symmetric top complex of heteronuclear diatomic molecules with a $C_{n h}(n \geq 3)$ symmetry is planar and obeys the relation: ${ }^{75}$

$$
D_{J K}+\frac{2}{3}\left(D_{J}+2 D_{K}\right)=0
$$

Fitted ground state distortion constants in Table 2 and the ab initio value of $D_{K}(0.98$ x $\left.10^{-6} \mathrm{~cm}^{-1}\right)$ have being substituted into the eq (5) which yields $(-1.3 \pm 4.0) \times 10^{-7} \mathrm{~cm}^{-1}$ and clearly shows that the resulting value is close to zero.

Secondly, we calculated the inertial defects of the complex in the ground and upper states from the following relation:

$$
\Delta=\frac{\hbar}{4 \pi c}\left(\frac{1}{C}-\frac{2}{B}\right)(6)
$$

From the ground state rotational constants reported in Table 2, the inertial defect is found to be equal to $-0.12(2) \mathrm{amu} \AA^{2}$, close enough to zero to be consistent with a planar cyclic geometry. For the excited states, the inertial defects show significant differences: for the inplane bending $v_{6}$ mode, $\Delta=0.04(1)$ amu $\AA^{2}$ and for the out-of-plane $v_{4}$ mode, $\Delta=-0.56(2)$ amu $\AA^{2}$. These results are quite consistent with the motions of the intermolecular bending mode. The inertial defect is close to zero in the $v_{6}$ mode excitation because the in-plane bending motion remains in the plane of the cyclic $(\mathrm{HF})_{3}$. On the other hand, the relatively high value of the inertial defect for the out-of-plane $v_{4}$ mode is consistent with a large amplitude motion out of plane of the trimer.

The rotational parameters derived from our spectroscopic fit clearly indicate that $(\mathrm{HF})_{3}$ is vibrationally averaged planar. The fitted constants can then be used to determine the center of mass separations $R_{C O M}$ of the HF constituents of the trimer, from the formula of the moment of inertia i.e.

$$
3 \mu_{F} R^{2}(H F)+M_{H F} R_{C O M}^{2}=\frac{h}{8 \pi^{2} c C}(7)
$$

We assumed that the intramolecular HF bond length remains unchanged with respect to the monomer ground state values, i.e. $R(H F)=0.926 \AA$. In any case, the distance $R_{C O M}$ is only slightly sensitive to the value of $R(H F)$ and does not degrade the precision in determining center of mass separations. In the same manner, we derived the separation in the 
$v_{6}$ and $v_{4}$ states from $C^{\prime}$ fitted constants. The small increase of about $1 \%$ observed in $R_{C O M}$ indicates a slight weakening of the hydrogen bond in the intermolecular states which is consistent with the negative sign of $\Delta B / B$ and $\Delta C / C$ experimental values, respectively equal to $2.1 \%$ and $1.9 \%$ for $v_{6}$ and $v_{4}$. Calculated rotational constants (Table 2) do not reach experimental accuracy but the rms deviation from experiment is smaller than $0.4 \%$ in the ground state and for $v_{6}$ and about $0.9 \%$ for $v_{4}$. Moreover, relative $\Delta B / B$ and $\Delta C / C$ calculated values are of same sign as experimental ones with similar variations ( $2 \%$ and $1.3 \%$ for $v_{6}$ and $v_{4}$, respectively). Table 3 gathers the vibrationally averaged center of mass separations and inertial defects for $(\mathrm{HF})_{3}$.

The narrow linewidths of the low frequency bending intermolecular transitions observed in $(\mathrm{HF})_{3}$ sharply contrast with the large broadenings evidenced in the donor acid stretch region of $(\mathrm{DF})_{3}{ }^{41}$ and $(\mathrm{HF})_{3}{ }^{36}$ This suggests significant longer lifetimes in the $\mathrm{v}_{6}=1$ and $\mathrm{v}_{4}=1$ upper states. The observation of these transition linewidths provides lower bounds for such excited statelifetime $\tau=1 / 2 \pi \Gamma$ where $\Gamma$ is the full width at half maximum (FWHM) of a transition. Experimental linewidths of 114(6) and 126(9) $\mathrm{MHz}$ are deduced from the spectra, for $v_{6}$ and $v_{4}$ respectively. Our experimental conditions are such that the observed linewidths are largely dominated by the instrumental width of the interferometer, equal to $0.0035 \mathrm{~cm}^{-1}(105 \mathrm{MHz})$ accounting for boxcar apodization. A deconvolution of several intense ${ }^{q} Q(J, K)$ transitions of the $v_{6}$ band with the boxcar function set to 105 MHzyields upper bounds of $70 \mathrm{MHz}$ for non-instrumental widths and consequently lower bounds of $2 \mathrm{~ns}$ for excited state $v_{6}$ and $v_{4}$ lifetimes, consistent with expectations for sub-Doppler, almost collisionless spectra of a low frequency mode in a stiff hydrogen-bonded system. Nevertheless our rough estimateis comparable to more accurate linewidths (74 MHz) measured for the ${ }^{q} P$ and ${ }^{q} R$ transitions of the librational band of $(\mathrm{HF})_{4}$ around $710 \mathrm{~cm}^{-}$ ${ }^{1}$ using a pulsed slit jet diode laser spectrometer. ${ }^{57}$

\section{6- Conclusion}

In the absence of permanent dipole moment and in the presence of extensive IVR homogeneous broadening in $(\mathrm{HF})_{3}$, we faced an unusual case where far infrared spectroscopy could provide structural information even for the ground state in contrast to microwave and MIR spectroscopies.

Taking advantage of the association between high resolution broadband FTS and jetcooled conditions, the Jet-AILES spectrum recorded in thefar infrared range at a rotational temperature of $30 \mathrm{~K}$ enabled to perform the simultaneous rotational analysis of two intermolecular $v_{6}$ and $v_{4}$ bending fundamentals of the cyclic HF trimer within the oblate symmetric rovibrator model. The 15 fitted molecular parameters, including band centers, rotational and quartic centrifugal distortion constants in both ground and upper states have been used to bring definite proofs to the vibrationally averaged planarity of the complex, (i) the consistency of the relationship between centrifugal distortion constants with the criterion for the planarity of the GS structure (ii) the expected value of inertia defects, very small in the GS and in the $\mathrm{v}_{6}=1$ state of the in-plane bending motion, and much larger in the $\mathrm{v}_{4}=1$ state of the large amplitude motion out of the plane of the trimer. On the other hand, the small 
increase of the center of mass separation of the HF constituents (GS value, $R_{C O M}=2.600(1)$ $\AA$ ) correlates well with the decrease of fitted rotational constantsupon excitation which indicates a slight weakening of the hydrogen bond in the bending states.

$\mathrm{Ab}$ initio vibrational analyses about cyclic $(\mathrm{HF})_{3}$ reproduced remarkably all the observed frequencies and gave an additional argument for the assignment of intermolecular bending fundamentals. It has been clearly proved that the shifts between experimental and computed frequencies previously obtained by a $6 \mathrm{D}$ variational approach are due to neglecting the coupling with stretching modes. Also they greatly contributed to the spectral analysis in providing reliable rotational values, namely about the $B$ rotational constant to start the initial prediction, and the $D_{K}$ quartic constant, kept fixed in the global fit with 15 free parameters. Finally, the assignment of several unidentified bands in the Jet-AILES MIR spectrum, correlated with the strongest absorption signal of cyclic $(\mathrm{HF})_{4}$, will be presented in a forthcoming paper.

\section{Acknowledgements}

The authors are grateful to SOLEIL and the AILES staff for providing technical support under the proposal 20110648. 


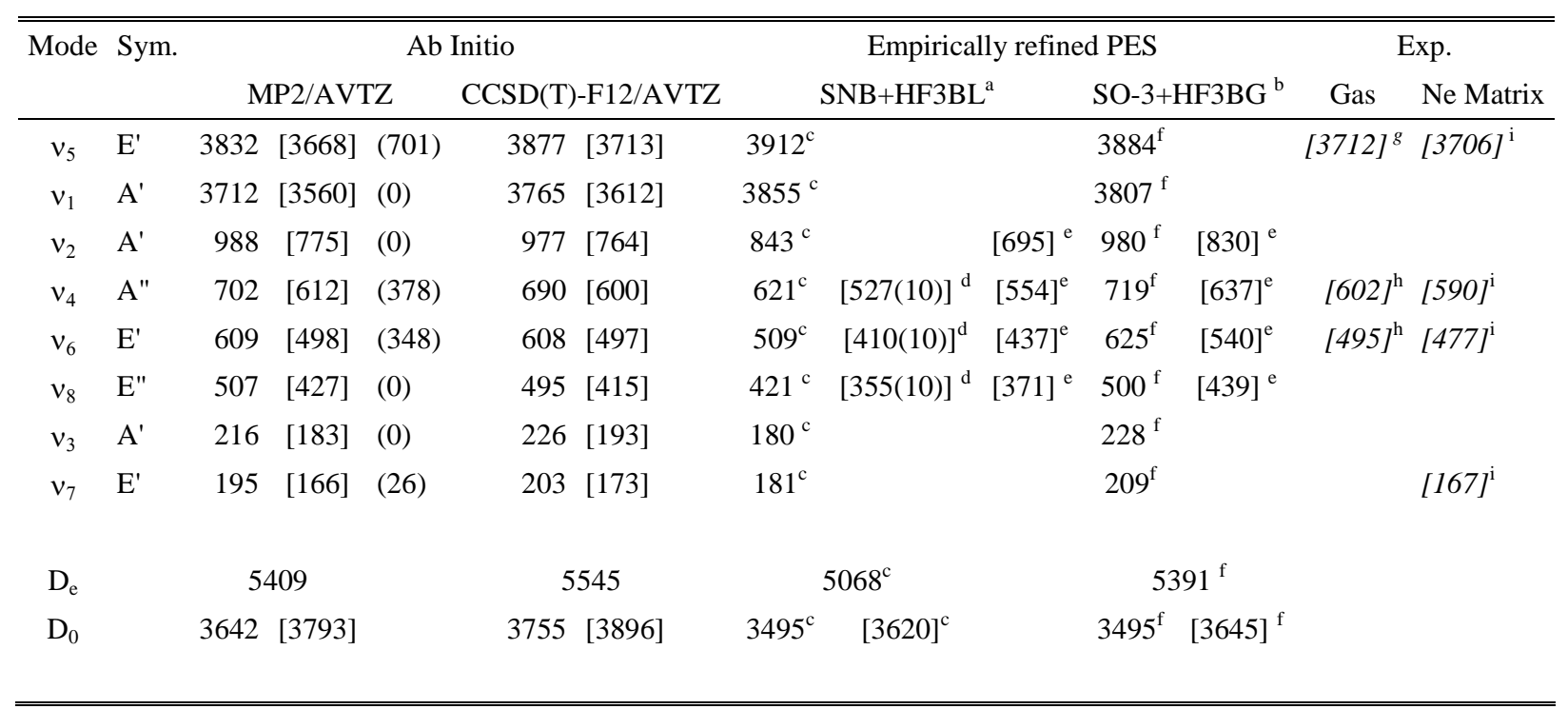

${ }^{\mathrm{a}} \operatorname{Ref}[76]$ and Ref [77].

${ }^{\mathrm{b}} \operatorname{Ref}[23]$ and Ref [77].

${ }^{\mathrm{c}} \operatorname{Ref}[76]$.

${ }^{\mathrm{d}}$ Approximate fully coupled DQMC calculations. Ref [76].

e $6 \mathrm{D}$ variational calculations. Ref [26].

${ }^{\mathrm{f}} \operatorname{Ref}[77]$.

${ }^{\mathrm{g}}$ Ref [36].

${ }^{\mathrm{h}}$ This work.

${ }^{\mathrm{i}} \operatorname{Ref}[48]$.

Table 1: Harmonic and [anharmonic] frequencies of cyclic $(\mathrm{HF})_{3}\left(\mathrm{~cm}^{-1}\right)$. Harmonic intensities are reported in parenthesis $(\mathrm{km} / \mathrm{mol}) . \mathrm{D}_{\mathrm{e}}$ and $\mathrm{D}_{0}$ energies with respect to $3 \mathrm{HF}$ molecules are also reported $\left(\mathrm{cm}^{-1}\right)$. 


\begin{tabular}{ccccccc}
\hline \multirow{2}{*}{ Parameter } & \multicolumn{2}{c}{ GS } & \multicolumn{2}{c}{$v_{6}$} & \multicolumn{2}{c}{$v_{4}$} \\
& Experiment & Ab initio & Experiment & Ab initio & Experiment & Ab initio \\
\hline$v_{0}$ & - & - & $494.76317(12)$ & $497^{b}$ & $602.42982(13)$ & $600^{b}$ \\
$\mathrm{~B}$ & $0.244608(7)$ & $0.24512^{b}$ & $0.239536(8)$ & $0.24026^{b}$ & $0.240462(7)$ & $0.24256^{b}$ \\
$\mathrm{C}$ & $0.122408(11)$ & $0.12211^{b}$ & $0.119738(11)$ & $0.11966^{b}$ & $0.120714(11)$ & $0.12020^{b}$ \\
$\mathrm{D}_{\mathrm{J}} \times 10^{6}$ & $2.01(3)$ & $1.49^{c}$ & $2.48(4)$ & - & $1.83(4)$ & - \\
$\mathrm{D}_{\mathrm{JK}} \times 10^{6}$ & $-2.77(4)$ & $-2.30^{c}$ & $-3.69(6)$ & - & $-2.23(6)$ & - \\
$\mathrm{D}_{\mathrm{K}} \times 10^{6}$ & $0.98^{\mathrm{a}}$ & $0.98^{c}$ & $1.47(3)$ & - & $0.71(3)$ & - \\
$\mathrm{J}, \mathrm{K}$ & - & - & 19,18 & - & 21,21 & - \\
\hline
\end{tabular}

${ }^{\mathrm{a}}$ Fixed to the ab initio value.

${ }^{\mathrm{b}} \mathrm{CCSD}(\mathrm{T})-\mathrm{F} 12 / \mathrm{AVTZ}$ results with anharmonic correction from VPT2 at MP2/AVTZ level of theory

${ }^{\mathrm{c}}$ VPT2 results at MP2/AVTZ level of theory.

Table 2: Molecular parameters $\left(\mathrm{cm}^{-1}\right)$ for the ground state, $v_{6}$ and $v_{4}$ states of cyclic $(\mathrm{HF})_{3}$.

\begin{tabular}{ccccc}
\hline & Equ. & GS & $v_{6}$ & $v_{4}$ \\
\hline $\mathrm{R}_{\mathrm{COM}}(\AA)$ & {$[2.551]$} & $2.600(1)[2.603]$ & $2.629(1)[2.630]$ & $2.619(1)[2.624]$ \\
\hline$\Delta\left(\mathrm{amu} \AA^{2}\right)$ & & $-0.12(2)$ & $0.04(1)$ & $-0.56(2)$ \\
\hline
\end{tabular}

Table 3: Vibrationally averaged HF's units center-of-mass separation and inertial defects for $(\mathrm{HF})_{3}$. CCSD(T)-F12/AVTZ values are reported in squared brackets. 


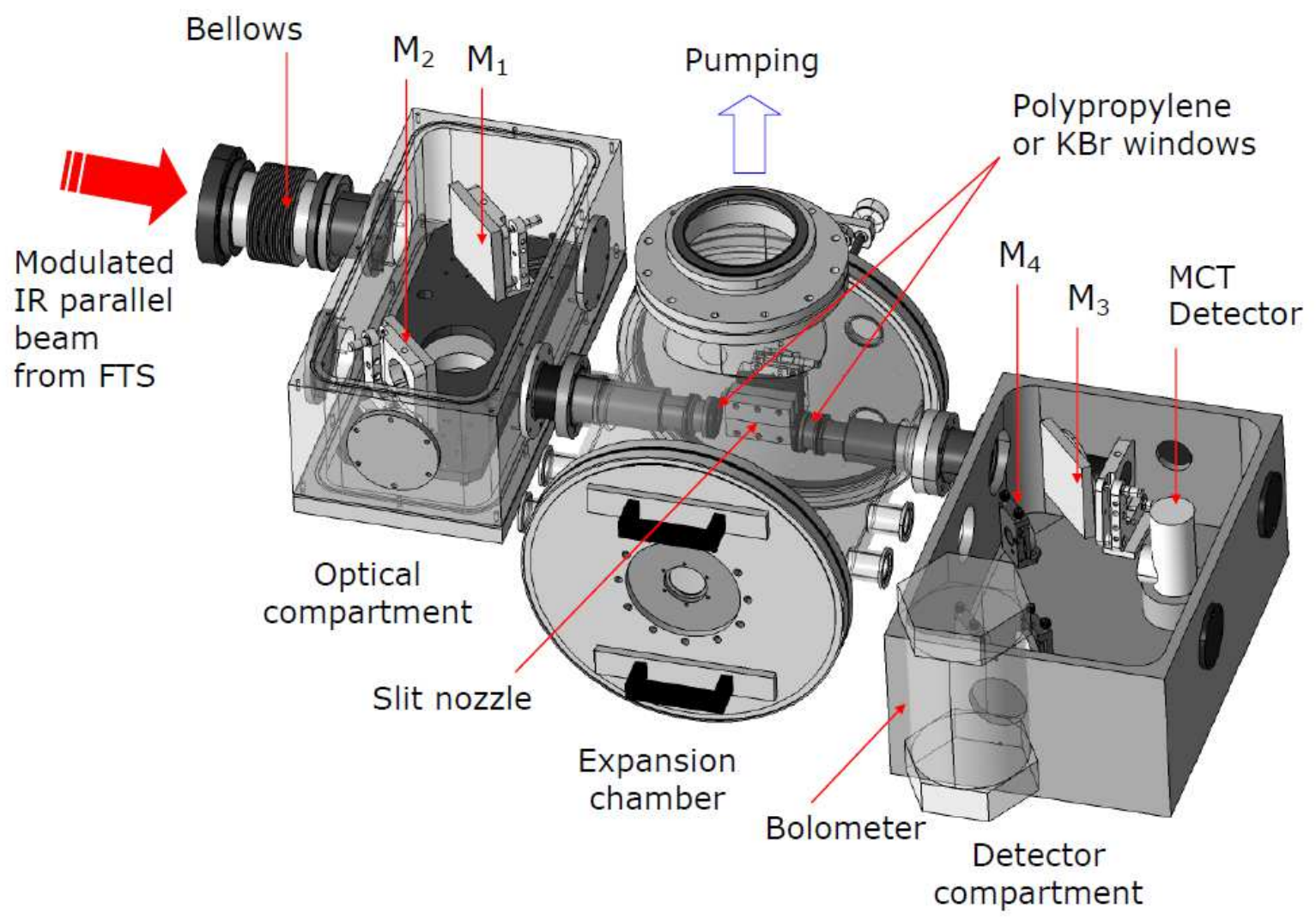

Figure 1: Schematic view of the Jet-AILES set-up. 


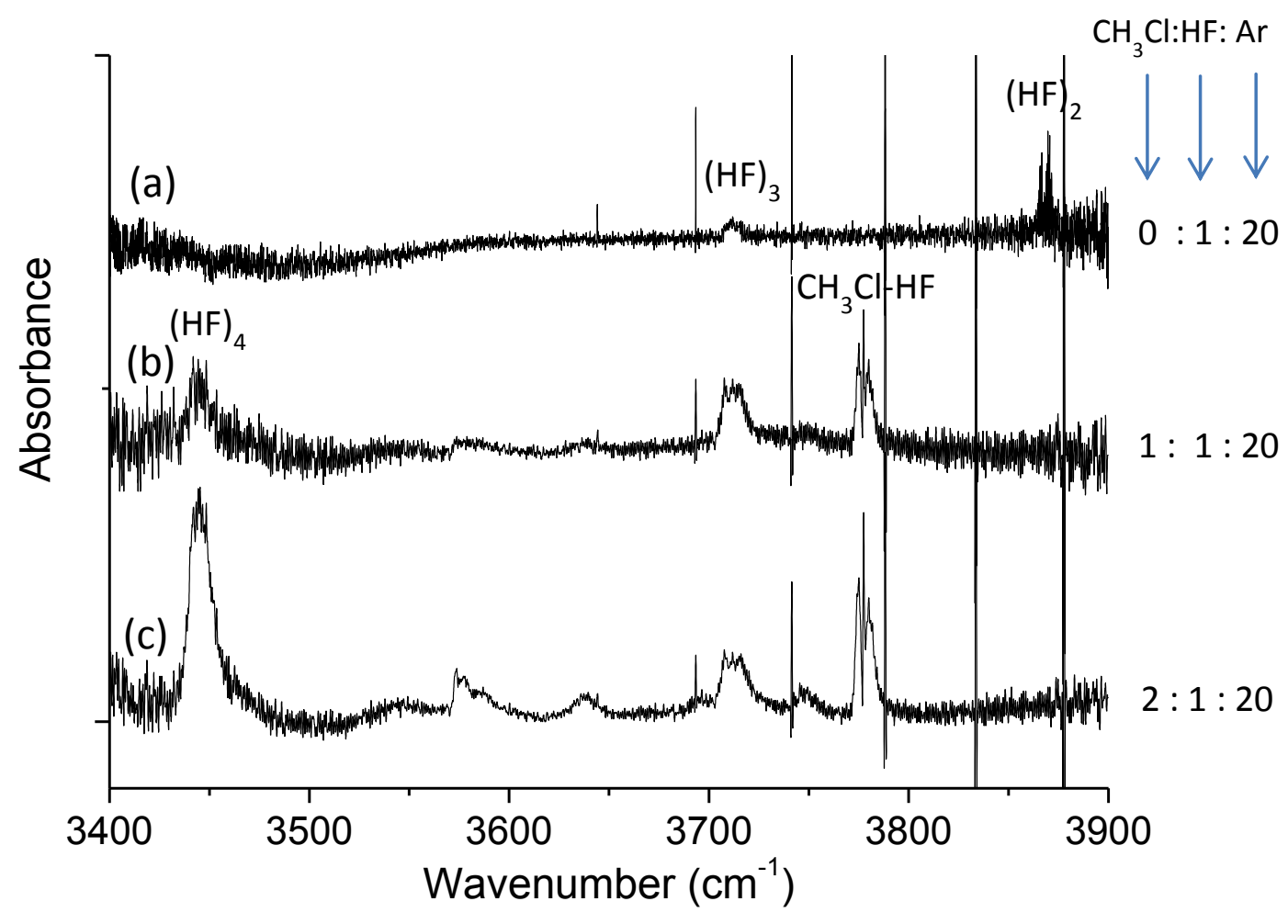

Figure 2: Jet-FTIR spectra of the HF stretch mode of small $(\mathrm{HF})_{\mathrm{n}}$ clusters recorded at $0.1 \mathrm{~cm}^{-1}$ resolution for different dilutions of the $\mathrm{CH}_{3} \mathrm{Cl}: \mathrm{HF}$ : $\mathrm{Ar}$ mixture. The intense and narrow bandsobserved in the $3700-3900 \mathrm{~cm}^{-1}$ range, separated by about $40 \mathrm{~cm}^{-1}$, are rotational $\mathrm{P}(\mathrm{J})$ lines of the HF monomer. 


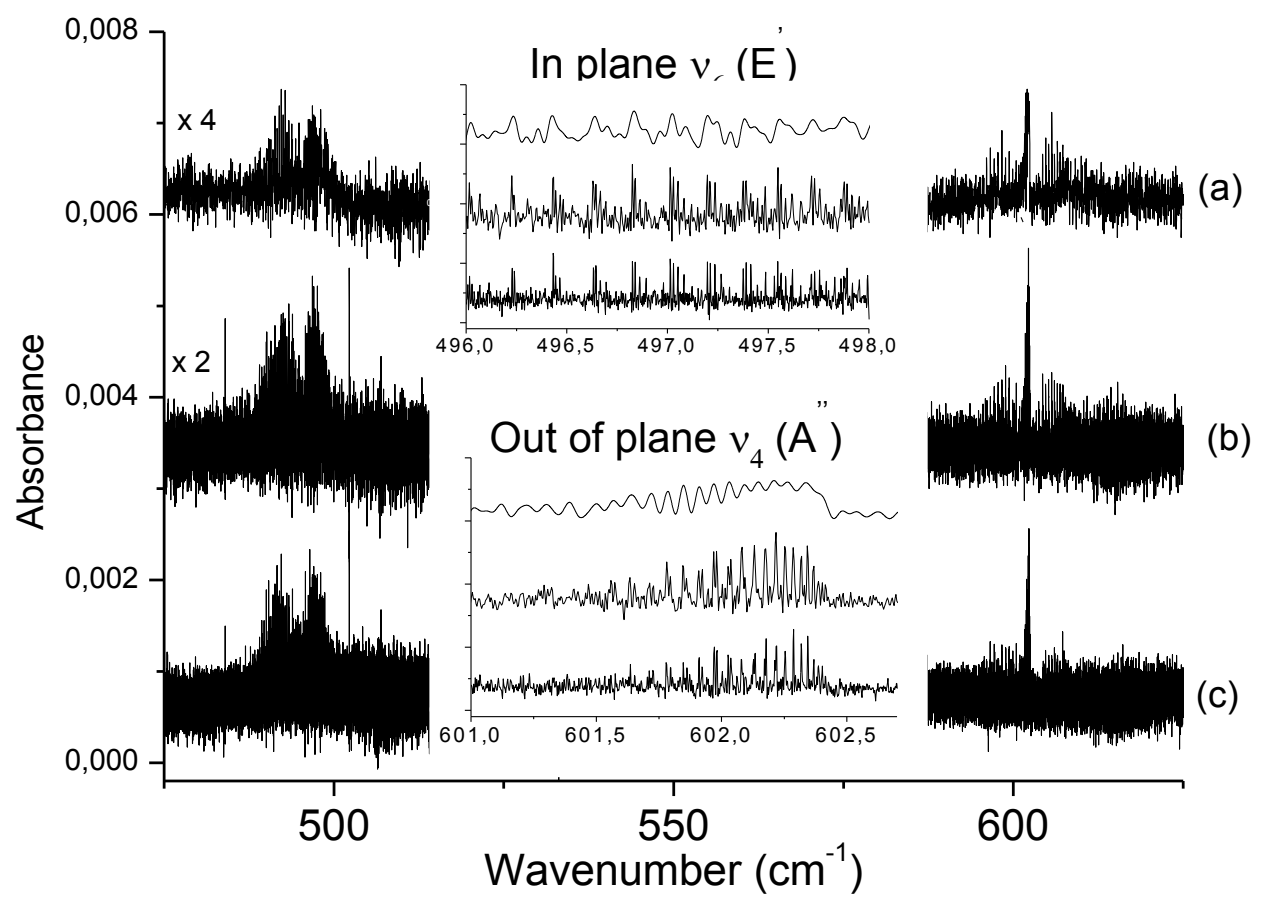

Figure 3: Jet-AILES FIR spectra of (HF) $)_{3}$ recorded at different resolutions: (a) 1000 scans at $0.05 \mathrm{~cm}^{-1}$, (b) 800 scans at $0.01 \mathrm{~cm}^{-1}$, (c) 700 scans at $0.005 \mathrm{~cm}^{-1}$. Two inserts clearly show the progressively appearance of the fully resolved rotational structure of the ${ }^{R} Q(J, K)$ branch of the perpendicular $v_{6}$ band (around $497 \mathrm{~cm}^{-1}$, see top panel) and of the ${ }^{Q} Q(J, K)$ branch of the parallel $v_{4}$ band (around $602 \mathrm{~cm}^{-1}$, see bottom panel) when improving the resolution. 


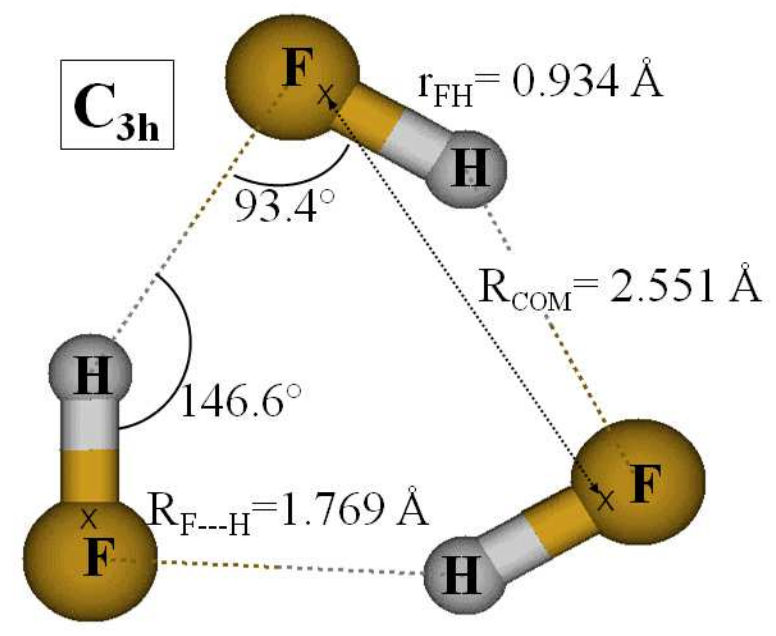

Figure 4:Equilibrium structure of $(\mathrm{HF})_{3}$ computed with CCSD(T)-F12/AVTZ. 


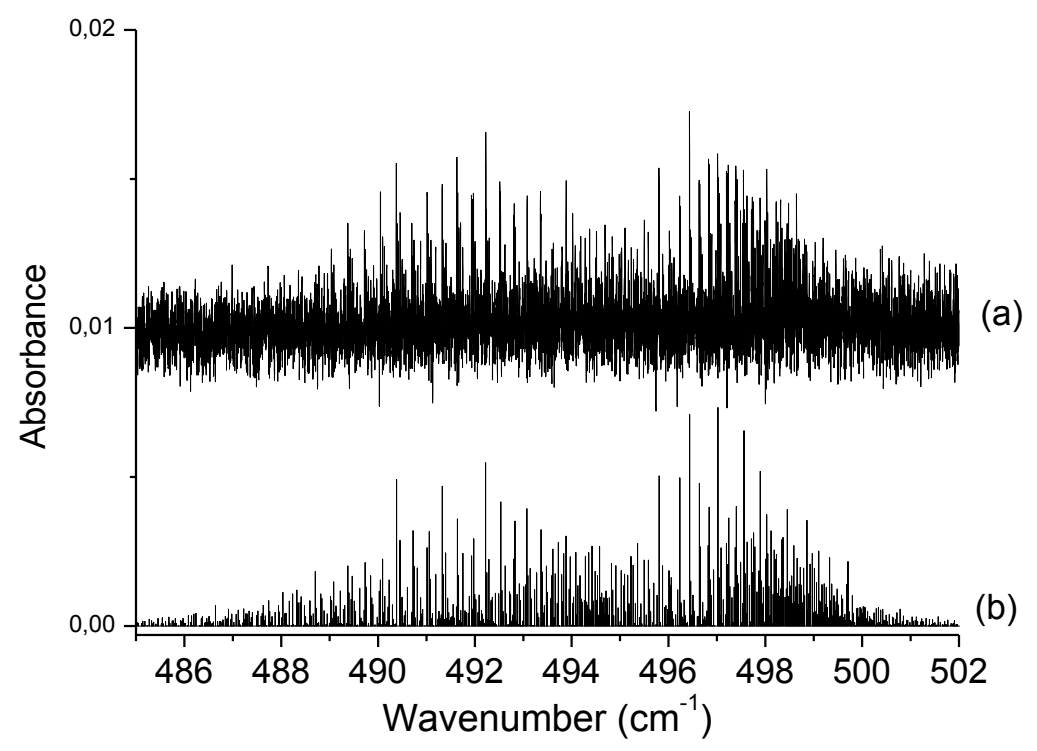

Figure 5: Global view of the Jet-AILES $v_{6}$ spectrum recorded at $0.005 \mathrm{~cm}^{-1}$ resolution (a) and our best simulation at $T_{\text {rot }}=30 \mathrm{~K}(\mathrm{~b})$. 


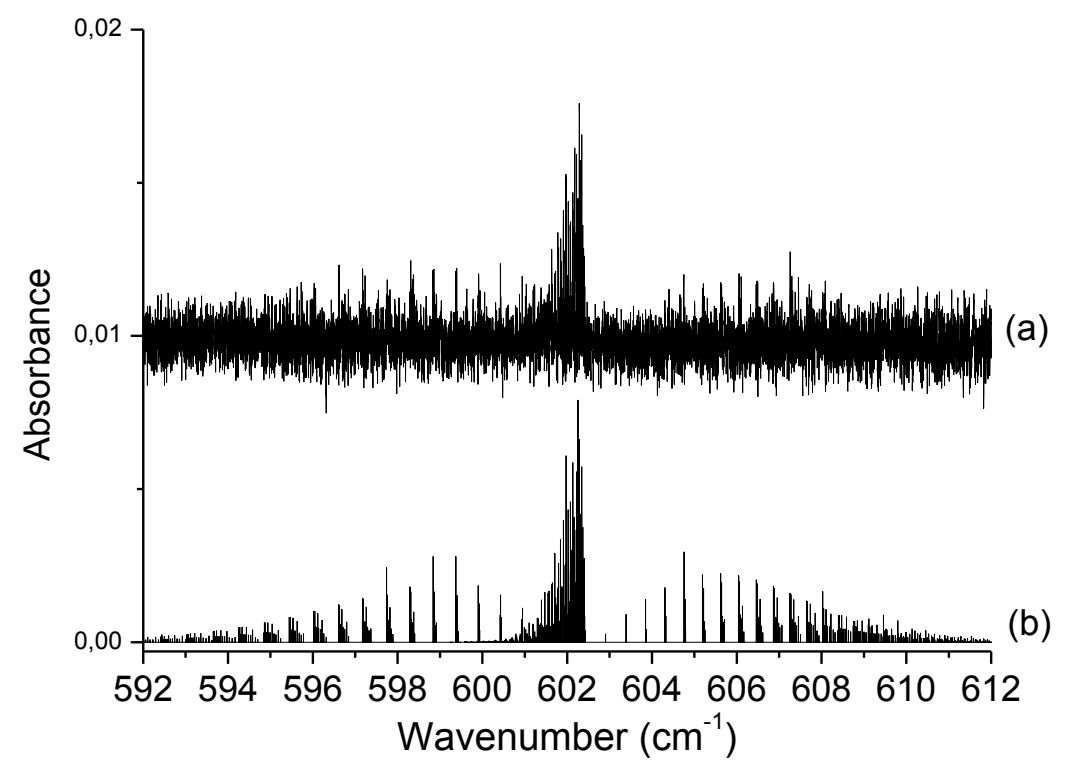

Figure 6: Global view of the Jet-AILES $v_{4}$ spectrum recorded at $0.005 \mathrm{~cm}^{-1}$ resolution (a) and our best simulation at $T_{\text {rot }}=30 \mathrm{~K}(\mathrm{~b})$. 


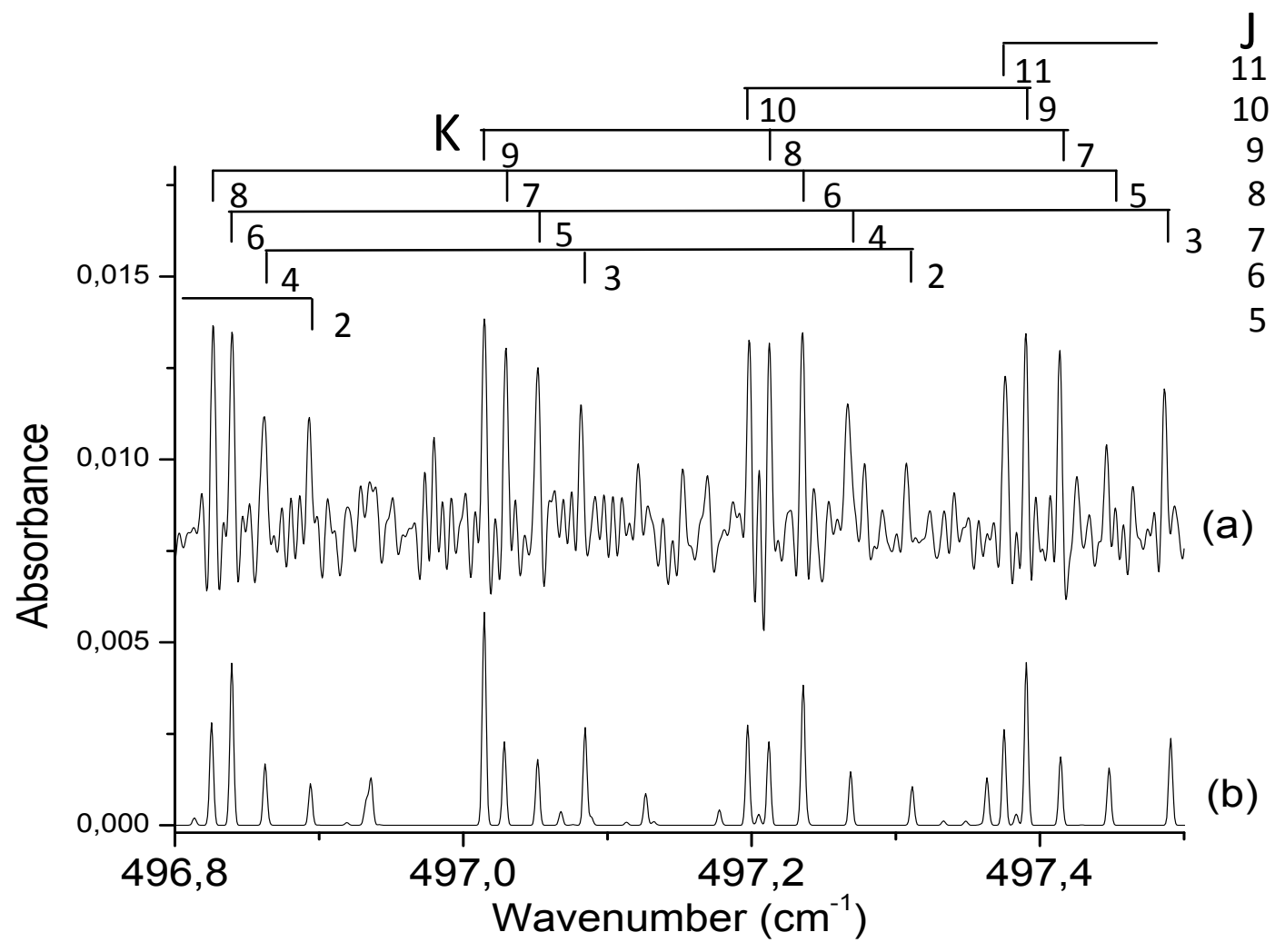

Figure 7: Portion of the ${ }^{r} R(J, K)$ branch of the bending in-plane $v_{6}$ band of cyclic $(\mathrm{HF})_{3}$ including assignments of the patterns ${ }^{r} R(J-n, K-2 n)$ : a) Jet-cooled spectrum recorded at $0.005 \mathrm{~cm}^{-1}$ resolution, (b) spectrum simulated at $T_{\text {rot }}=30 \mathrm{~K}$. 


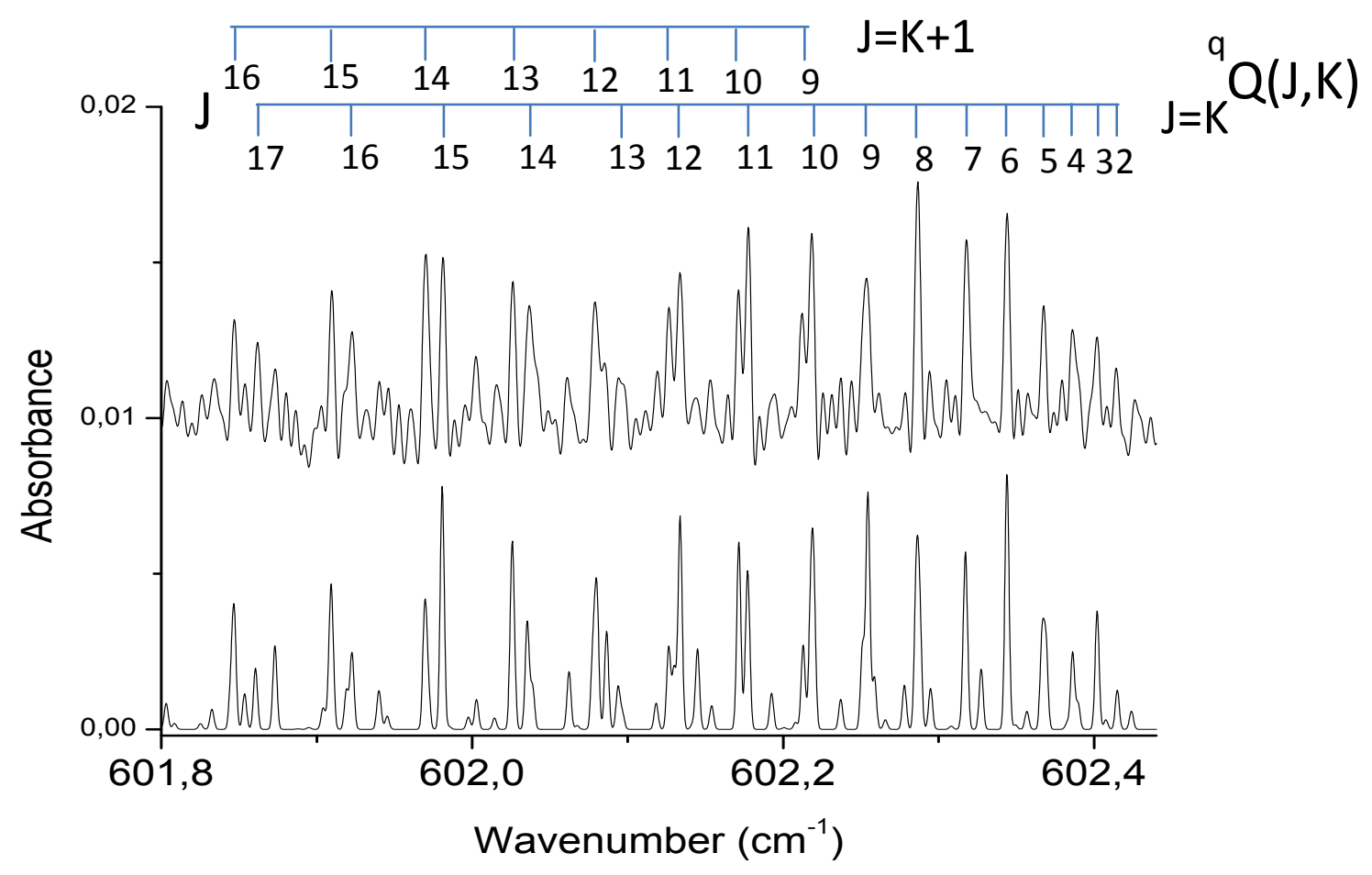

Figure 8: Portion of the ${ }^{q} Q(J, K)$ branch of the bending out-of-plane $v_{4}$ band of cyclic $(\mathrm{HF})_{3}$ including assignments of the patterns with $J=K$ and $J=K+1$ : a) Jet-cooled spectrum recorded at $0.005 \mathrm{~cm}^{-1}$ resolution, (b) spectrum simulated at $T_{\text {rot }}=30 \mathrm{~K}$. 


\section{References}

${ }^{1}$ M. A. Suhm and D. J. Nesbitt, Chem. Soc. Rev. 1995, 24, 45.

${ }^{2}$ D.J. Nesbitt, Chem. Rev. 1988, 88, 843.

${ }^{3}$ R.E. Miller, Acc. Chem. Res. 1991, 23, 10.

${ }^{4}$ M. Quack and M. A. Suhm, J. Chem. Phys. 1991, 95, 28.

${ }^{5}$ R.C. Cohen, and R. J. Saykally, J. Phys.Chem.1992, 96, 1024.

${ }^{6}$ G. Chalasinski, S. M. Cybulski, M. M. Szczesniak and S. Scheiner, J. Chem. Phys. 1989, 91, 7048.

${ }^{7}$ A. Karpfen, A. Beyer, and P. Schuster, Chem. Phys. Lett. 1983, 102, 289.

${ }^{8}$ S. Y. Liu, D. W. Michael, C. E. Dykstra, and J. W. Lisy, J. Chem. Phys. 1986, 84, 5032.

${ }^{9}$ K. D. Kolenbrander, C. E. Dykstra and J. M. Lisy, J. Chem. Phys. 1988, 88, 5995.

${ }^{10}$ C. E. Dykstra, Acc. Chem. Res. 1988, 21, 356.

${ }^{11}$ C. Zhang, D.L. Freeman and J.D. Doll, J. Phys.Chem.1989, 91, 2489.

${ }^{12}$ C. E. Dykstra, J. Phys.Chem.1990, 94, 180.

${ }^{13}$ A. Karpfen and O. Yanovitskii, J. Mol. Struct. 1994, 307, 81.

${ }^{14}$ M. J. Elrod and R. J. Saykally, Chem. Rev. 1994, 94, 1975.

${ }^{15}$ F. Huisken, E. G. Tarakanova, A. A. Vigasin, and G. V. Yukhnevich, Chem. Phys. Lett. 1995, 245, 319.

${ }^{16}$ K. R. Liedl, R. T. Kroemer and B. M. Rode, Chem. Phys. Lett. 1995, 246, 455.

${ }^{17}$ C. Maerker, P. R. Schleyer, K. R. Liedl, T. K. Ha, M. Quack, and M. A. Suhm, J. Comput. Chem, 1997, 18, 1695.

${ }^{18}$ G. S. Tschumper, Y. Yamaguchi, and H.F. Schaefer, J. Chem. Phys. 1997, 106, 9627.

${ }^{19}$ F. Huisken, M. Kaloudis and A. A. Vigasin, Chem. Phys. Lett. 1997, 269, 235.

${ }^{20}$ K. R. Liedl, S. Sekusak, R. T. Kroemer and B. M. Rode, J. Phys. Chem. A 1997, 101, 4707.

${ }^{21}$ M. P. Hodges, A. J. Stone, and E. C. Lago, J. Phys.Chem. A 1998, 102, 2455.

${ }^{22}$ K. R. Liedl, R. T. Kroemer,J. Phys.Chem. A 1998, 102, 1832.

${ }^{23}$ W. Klopper, M. Quack, and M. A. Suhm, J. Chem. Phys. 1998, 108, 10096.

${ }^{24}$ W. Klopper, M. Quack, and M. A. Suhm, Mol. Phys. 1998, 94, 105.

${ }^{25}$ B. L. Grigorenko, A. A. Moskovsky and A. V. Nemukhin, J. Chem. Phys. 1999, 111, 4442.

${ }^{26}$ X-G. Wang and T. Carrington, J. Chem. Phys. 2001, 115, 9781.;X-G. Wang and T. Carrington, J. Chem. Phys. 2003, 119, 12682.

${ }^{27}$ T. R. Dyke, B. J. Howard and W. Klemperer, J. Chem. Phys. 1972, 56, 2442.

${ }^{28}$ B. J. Howard, T. R. Dyke and W. Klemperer, J. Chem. Phys. 1984, 81, 5417.

${ }^{29}$ W. J. Lafferty, R. D. Suenram, and F. J. Lovas, J. Mol. Spec. 1987, 123, 434.

${ }^{30}$ A. S. Pine and W. J. Lafferty, J. Chem. Phys. 1983, 78, 2154.

${ }^{31}$ A. S. Pine,W. J. Lafferty and B. J. Howard , J. Chem. Phys. 1984, 81, 2939.

${ }^{32}$ A. S. Pine, and B. J. Howard , J. Chem. Phys. 1986, 84, 590.

${ }^{33}$ D. T. Anderson, S. Davis and D. J. Nesbitt, J. Chem. Phys. 1996, 105, 6645.

${ }^{34}$ J. M. Lisy, A. Tramer, M. F. Vernon and Y. T. Lee, J. Chem. Phys. 1981, 75, 4733.

${ }^{35}$ M. F. Vernon, J. M. Lisy, D. J. Krajnovich, A. Tramer, H-S. Kwok, Y. R. Shen and YT. Lee, Faraday Discuss. Chem. Soc. 1982,73, 387.

${ }^{36}$ D. W. Michael and J. M. Lisy, J. Chem. Phys. 1986, 85, 2528.

${ }^{37}$ K. von Puttkamer and M. Quack, Mol. Phys. 1987, 62, 1047.

${ }^{38}$ K. von Puttkamer and M. Quack, Chem. Phys. 1989, 139, 31.

${ }^{39}$ M. Quack and M. A. Suhm, Chem. Phys. Lett. 1990, 171, 517.

${ }^{40}$ M. A. Suhm, J. T. Farrell, A. McLiroy and D. J. Nesbitt, J. Chem. Phys. 1992, 97, 5341.

${ }^{41}$ M. A. Suhm, J. T. Farrell, S. H. Ashworth and D. J. Nesbitt, J. Chem. Phys. 1993, 98, 5985.

${ }^{42}$ M. Quack, U. Schmitt and M. A. Suhm, Chem. Phys. Lett. 1993, 208, 446.

${ }^{43}$ F. Huisken, M. Kaloudis, A. Kulcke and D. Voelkel, Infrared Phys. Technol. 1995, 36, 171. 
${ }^{44}$ F. Huisken, M. Kaloudis, A. Kulcke, C. Lausch and J. M. Lisy, J. Chem. Phys. 1995, 103, 5366.

${ }^{45}$ D. Luckhaus, M. Quack, U. Schmitt and M. A. Suhm, Ber. Bunsenges Phys. Chem. 1995, 99, 457.

${ }^{46}$ M. Quack, U. Schmitt and M. A. Suhm, Chem. Phys. Lett. 1997, 269, 29.

${ }^{47}$ L. Andrews, V. E. Bondibey and J. H. English, J. Chem. Phys. 1984, 81, 3425.

${ }^{48}$ L. Andrews, S. R. Davis and R. D. Hunt, Mol. Phys. 1992, 77, 993.

${ }^{49}$ G. C. Hancock, D. G. Truhlar and C. E. Dykstra, J. Chem. Phys. 1988, 88, 1786.

${ }^{50}$ P. R. Bunker, M. Kofranek, H. Lischka, and A. Karpfen, J. Chem. Phys. 1988, 89, 3002.

${ }^{51}$ D. Prichard, J. S. Muenter and B. J. Howard, Chem. Phys. Lett. 1987, 135, 9.

${ }^{52}$ K. W. Jucks and R. E. Miller, J. Chem. Phys. 1988, 88, 2196.

${ }^{53}$ J. Han, Z. Wang, A. L. McIntosh, R. R. Lucchese and J. W. Bevan, J. Chem. Phys. 1994, 100, 7101.

${ }^{54}$ G. T. Fraser, A. S. Pine, W. J. Lafferty, and R. E. Miller, J. Chem. Phys. 1987, 87, 1502.

${ }^{55}$ M. J. Weida, J. M. Sperhac and D. J. Nesbitt, J. Chem. Phys. 1995, 103, 7685.

${ }^{56}$ N. Pugliano, and R. J. Saykally, Science, 1992, 257, 1937.

${ }^{57}$ T. H. Blake, S. W. Sharpe and S. S. Xantheas, J. Chem. Phys. 2000, 113, 707.

${ }^{58}$ M. A. Suhm and F. Kollipost, Phys. Chem. Chem. Phys. 2013, 13, 811.

${ }^{59}$ M. Cirtog, P. Asselin, P. Soulard, B. Tremblay, B. Madebène,M.E. Alikhani, R. Georges, A. Moudens, M. Goubet, T.R.Huet, et al., J. Phys. Chem. A 2011, 115, 2523.

${ }^{60}$ O. Pirali, M. Goubet, T. R. Huet, R. Georges, P. Soulard, P. Asselin, J. Courbe,P. Roy and M. Vervloet, Phys. Chem. Chem. Phys. 2013,15, 10141.

${ }^{61}$ V. Boudon, P. Asselin, P. Soulard, M. Goubet, T.R. Huet, R. Georges, O. Pirali and P. Roy, Mol. Phys. 2013, DOI:10.1080/00268976.2013.793888.

${ }^{62}$ C. M. Lovejoy and D. J. Nesbitt, J. Chem. Phys. 1989, 90, 4671.

${ }^{63}$ M. J. Frisch et al. Gaussian 09, Revision A.02, Gaussian, Inc.: Wallingford, CT, 2009.

${ }^{64}$ H.-J.Werner andP. J.Knowles, MOLPRO, a package of ab initio programs; Cardiff University: Cardiff, U.K., 2012.

${ }^{65}$ T. H. Jr.Dunning, J. Chem. Phys. 1989, 90, 1007.

${ }^{66}$ R. A. Kendall, T. H. Jr. Dunning and R. J.Harrison, J. Chem. Phys. 1992,96, 6796.

${ }^{67}$ V.Barone, J. Chem. Phys. 2005,122, 14108.

${ }^{68}$ S. S. Xantheas, Int. Rev. Phys. Chem. 2006, 25, 719.

${ }^{69}$ G. M. Chaban and R. B. Gerber, Spectrochim. Acta A 2002, 58, 887.

${ }^{70}$ B. Tremblay, B. Madebène, M. E. Alikhani and J.P. Perchard, Chem. Phys., 2010, 378, 27.

${ }^{71}$ G. Herzberg, Molecular Spectra and Molecular Structure II, Infrared and Raman Spectra, D. Van Nostrand Company, Inc.,New York, 1945.

${ }^{72}$ A. Weber, J. Chem. Phys. 1988, 88, 3428.

${ }^{73}$ H. M. Pickett, J. Mol. Spec. 1991, 148, 371.

${ }^{74}$ PGOPHER, a program for Simulating Rotational Structure, C. M. Western, University of Bristol, http://pgopher.chm.bris.ac.uk

${ }^{75}$ J. M. Dowling, J. Mol. Spec. 1961, 6, 550.

${ }^{76}$ M. A. Suhm, HF Dampf. Habilitationsschrift, ETH Zürich 1995.

${ }^{77}$ M. Quack, J. Stohner and M. A. Suhm, J. Mol. Struct. 2001, 599, 381. 